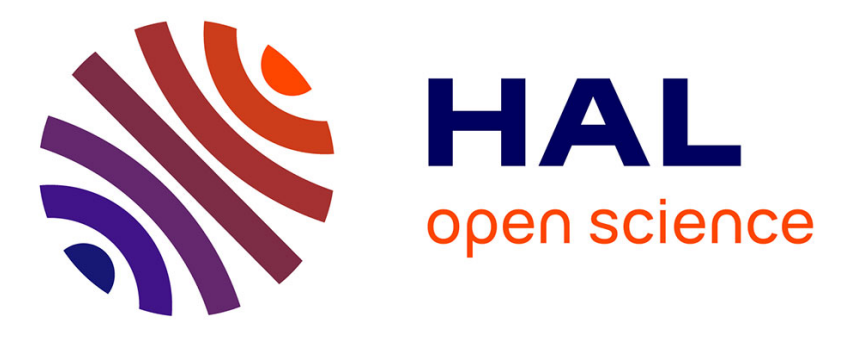

\title{
Acyclic orientations with path constraints
}

\author{
Rosa Figueiredo, Valmir C Barbosa, Nelson Maculan, Cid C de Souza
}

\section{To cite this version:}

Rosa Figueiredo, Valmir C Barbosa, Nelson Maculan, Cid C de Souza. Acyclic orientations with path constraints. RAIRO - Operations Research, 2009, 42 (4), pp.455-467. 10.1051/ro:2008028 . hal-02184964

\section{HAL Id: hal-02184964 \\ https://hal.science/hal-02184964}

Submitted on 16 Jul 2019

HAL is a multi-disciplinary open access archive for the deposit and dissemination of scientific research documents, whether they are published or not. The documents may come from teaching and research institutions in France or abroad, or from public or private research centers.
L'archive ouverte pluridisciplinaire HAL, est destinée au dépôt et à la diffusion de documents scientifiques de niveau recherche, publiés ou non, émanant des établissements d'enseignement et de recherche français ou étrangers, des laboratoires publics ou privés. 


\title{
RAIRO Operations Research
}

Will be set by the publisher

\section{ACYCLIC ORIENTATIONS WITH PATH CONSTRAINTS*}

\author{
Rosa M. V. Figueiredo ${ }^{1}$, Valmir C. Barbosa ${ }^{2}$, \\ Nelson MaCulan ${ }^{2}$ and Cid C. DE Souza ${ }^{3}$
}

\begin{abstract}
Many well-known combinatorial optimization problems can be stated over the set of acyclic orientations of an undirected graph. For example, acyclic orientations with certain diameter constraints are closely related to the optimal solutions of the vertex coloring and frequency assignment problems. In this paper we introduce a linear programming formulation of acyclic orientations with path constraints, and discuss its use in the solution of the vertex coloring problem and some versions of the frequency assignment problem. A study of the polytope associated with the formulation is presented, including proofs of which constraints of the formulation are facet-defining and the introduction of new classes of valid inequalities.
\end{abstract}

Keywords: Acyclic orientations, path constraints, combinatorial optimization problems, facets of polyhedra

Mathematics Subject Classification. 90C27, 90C57

\section{INTRODUCTION}

Let $G=(V, E)$ be an undirected graph, $V$ its set of vertices, and $E$ its set of edges. An orientation of $G$ is a function $\omega$ with domain $E$ such that $\omega([i, j]) \in$

May 11, 2007

* The authors acknowledge partial support from CNPq, CAPES, and a FAPERJ BBP grant.

${ }^{1}$ Universidade do Estado do Rio de Janeiro, Instituto de Matemática e Estatística, 20550-900 Rio de Janeiro - RJ, Brazil; e-mail: rosa@ime.uerj.br

2 Universidade Federal do Rio de Janeiro, Programa de Engenharia de Sistemas e Computação, COPPE, Caixa Postal 68511, 21941-972 Rio de Janeiro - RJ, Brazil; e-mail: valmir@cos.ufrj.br \& maculan@cos.ufrj.br

${ }^{3}$ Universidade Estadual de Campinas, Instituto de Computação, Caixa Postal 6176, 13084971 Campinas - SP, Brazil; e-mail: cid@ic.unicamp.br 
$\{(i, j),(j, i)\}$ for all $[i, j] \in E$. That is, $\omega$ assigns a direction to each edge in $E$. Given an orientation $\omega$, let $G_{\omega}$ be the directed graph obtained by replacing each edge $[i, j] \in E$ with the arc $\omega([i, j])$. An orientation is said to be acyclic if $G_{\omega}$ contains no directed cycle.

Many combinatorial optimization problems can be solved by determining an optimal orientation of a graph with respect to some measure of optimality. One example is the linear ordering problem [7], also called the permutation problem or the triangulation problem, and closely related to the acyclic subdigraph problem [8]. Given a complete directed graph $D_{n}=\left(V_{n}, A_{n}\right)$ on $n$ vertices and arc weights $c_{i j}$ for each arc $(i, j) \in A_{n}$, the linear ordering problem consists in finding a spanning acyclic tournament in $D_{n}$ such that the sum of the weights of its arcs is as large as possible. The spanning acyclic tournament in $D_{n}$ is equivalent to an acyclic orientation of the complete undirected graph with $n$ vertices.

Another problem whose solution is given by an optimal orientation is discussed in [2]. Given an undirected graph $G$ and an orientation $\omega$, not necessarily acyclic, the distance $\operatorname{dist}(i, j)$ from a vertex $i$ to another vertex $j$ is the length of the shortest path from $i$ to $j$ in $G_{\omega}$. The diameter of $G_{\omega}$ is defined in [2] as $\max _{i, j \in V} \operatorname{dist}(i, j)$. An annular network is a graph that can be represented as a two-dimensional grid consisting of a number of concentric circles around a center and some straight lines crossing all the circles. The problem of finding an orientation of minimum diameter when $G$ is an annular network is useful in various applications [2].

A communications network can be modeled as the undirected graph $G$ if vertices represent processors and edges communications links between pairs of processors. Each vertex in $V$ must be assigned a set of buffers in order to store messages which move through the network. For every network there exists a lower bound on the number of buffers which have to be maintained at each vertex to allow deadlockfree routing. In [3] an approach to prevent deadlocks is investigated in which finding the minimum number of buffers is related to finding an optimal acyclic orientation $\omega$ of $G$. The optimality criterion used in this case is to minimize the maximum number of changes of orientations on some directed paths in $G_{\omega}$.

Knowledge about acyclic orientations can also be useful in the solution of the vertex coloring problem. Roy [10] and Gallai [6], independently, showed that, given an undirected graph $G$, the length of a longest elementary path of each possible orientation of $G$ yields an upper bound on the chromatic number of $G$. Also, they proved that the exact value of the chromatic number is accomplished by the minimum bound over the set of all orientations of $G$. In a subsequent work [5], Deming showed that it is sufficient to consider just the set of acyclic orientations of $G$. In the same work, Deming also showed that using another measure of optimality, again over the set of the acyclic orientations of $G$, it is possible to find the maximum independent set of that graph. In this case, and for $\omega$ an acyclic orientation, the criterion is to maximize the size of a minimum chain decomposition of $G_{\omega}$.

Since frequency assignment problems are closely related to the vertex coloring problem, it would be expected that an orientation-based approach could also be 
proposed for that problem. This is done in [4], where a two-stage integer programming model is proposed in which the outer stage consists of an acyclic subdigraph problem with additional longest-path constraints. Also, in [9] an orientation model is pointed at as a promising way to obtain good lower bounds to be incorporated into a metaheuristic approach to the frequency assignment problem.

All these applications have motivated us to the study of the acyclic orientations of an undirected graph. In the present study, we introduce an integer programming formulation of the set of all acyclic orientations with constraints defined over a set of paths. As we demonstrate in Section 1, there are well-known combinatorial optimization problems that relate clearly to acyclic orientations with path constraints.

We close this section by giving the necessary definitions and notations to be used throughout the paper. Let $G=(V, E)$ be an undirected graph. A coloring of $G$ is an assignment of labels to each vertex such that the end-vertices of any edge have different labels. The chromatic number of $G$ is the smallest number of different labels needed to define a coloring of $G$ and is denoted by $\chi(G)$. The vertex coloring problem is the problem of providing $G$ with a coloring that employs $\chi(G)$ labels. Let $D=(V, A)$ be a digraph. If $D$ is an acyclic digraph, the diameter of $D$ with respect to a vector $c \in \mathbb{R}^{|A|}$ of arc weights is the length of a longest weighted path in $D$. Given an arc set $B \subseteq A$, we denote by $D[B]$ the subdigraph of $D$ induced by $B$. If $D[B]$ is an acyclic digraph, then we say that $B$ is an acyclic arc set of $D$. For the sake of conciseness, we henceforth use path to refer to an elementary path and equate a path with its arc set.

The remainder of the paper is structured as follows. In Section 1 we relate the vertex coloring and frequency assignment problems to orientations with diameter constraints. A model of acyclic orientations with another kind of path constraints is presented in Section 2. We prove that this model can also be used to find an orientation with diameter constraints. In Section 3 we investigate the polyhedral structure of the polytope associated with the model proposed. Finally, in Section 4 we discuss directions for further investigation.

\section{Combinatorial optimization problems and aCyCliC ORIENTATIONS WITH DIAMETER CONSTRAINTS}

As noted above, in $[6,10]$ the chromatic number $\chi(G)$ of an undirected graph $G$ is described as an optimization problem over the set of all orientations of $G$. This result is revised in [5], where the author proves that it is sufficient to consider only the set of acyclic orientations, that is,

$$
\chi(G)=1+\min _{\omega \in \Omega} \max _{p \in \mathcal{P}_{\omega}}|p|,
$$

where $\Omega$ denotes the set of all acyclic orientations of $G, \mathcal{P}_{\omega}$ the set of paths in $G_{\omega}$, and $|p|$ the number of arcs in $p$. Alternatively, (1) asks for $\omega$ such that $G_{\omega}$ has the least possible diameter considering arc weights equal to 1 , which is then $\chi(G)-1$. 
Let $\omega^{*}$ be an acyclic orientation that solves (1). The source decomposition of the graph $G_{\omega^{*}}$ gives us, in polynomial time, a coloring of $G$ that uses exactly $\chi(G)$ labels. An acyclic directed graph has a unique source decomposition defined as a partition of the vertex set $V$ into sets $V_{1}, V_{2}, \ldots, V_{k}$, where $V_{i}$ is the set of all vertices of the subgraph induced by $V \backslash\left\{V_{1} \cup \cdots \cup V_{i-1}\right\}$ whose in-degree is zero. Notice that each vertex set $V_{i}$ in the partition $\left(V_{1}, V_{2}, \ldots, V_{k}\right)$ is an independent set, and then the partition defines a coloring of $G$. Then, in order to solve the vertex coloring problem for a graph $G$ it is sufficient to find an acyclic orientation $G_{\omega^{*}}$ with minimum diameter. Henceforth, we let $q(G)=\chi(G)-1$ denote the length of the minimum diameter over all acyclic orientations of $G$.

In the literature, different problems can be found under the common heading of frequency assignment problem (FAP). In all of them, the classic approach relates the problem to the vertex coloring problem. It then follows from our previous discussion that we can consider using acyclic orientations also to solve this problem. This is done in [4], where a new orientation model is proposed for a version of FAP, and in [9], where the LP bounds of an orientation model are suggested to be incorporated into a metaheuristic proposed for another version of FAP. In order to describe the idea of an orientation model for FAP, we next introduce the elements usually found in the definition of that problem. Let $\mathcal{L}$ denote an index set of links, $\mathcal{F}_{i}$ a set of available frequencies for link $i \in \mathcal{L}$, and $d_{i j}$ a channel separation that defines the minimum distance between frequencies assigned to links $i, j \in \mathcal{L}$. The inexistence of a channel separation between links $i$ and $j$ can be imposed by setting $d_{i j}=0$. A frequency assignment specifies, for each $i \in \mathcal{L}$, a frequency in $\mathcal{F}_{i}$ for link $i$.

The most common version of FAP asks for an assignment of frequencies that minimizes the number of frequencies used, known as the frequency spectrum, while satisfying the channel separation imposed by $d_{i j}$ for all $i, j \in \mathcal{L}$. Clearly, when every $d_{i j}$ is equal to zero or one and, for each $i \in \mathcal{L}, F_{i}=\mathcal{L}$, then FAP can be easily cast into the vertex coloring problem and can therefore be solved by looking for an acyclic orientation of minimum diameter. In other FAP versions, given a fixed frequency spectrum $\Phi$ we must find an assignment of frequencies to the links that minimizes a cost function, sometimes an interference function, defined over the channel separation constraints. Next we describe the orientation model presented in [9] for one of these versions of FAP.

We use a graph $G=(V, E)$ with $V$ being the set of links $\mathcal{L}$. A pair of vertices $i, j \in V$ is connected by an edge $[i, j] \in E$ if and only if there is a distance requirement imposed by a channel separation $d_{i j}>0$ on the frequencies that are to be assigned to $i$ and $j$. Let $f_{i}$ be a positive integer variable specifying the frequency assigned to link $i, \zeta_{i j}$ a binary variable which is equal to 1 if and only if the channel separation between $f_{i}$ and $f_{j}$ is not guaranteed, and $c_{i j}$ the cost of violating the channel separation constraint. In order to specify an orientation of edge $[i, j] \in E$, a binary decision variable $o_{i j}$ is introduced and defined as follows: $o_{i j}=1$ if edge $[i, j] \in E$ is oriented from $i$ to $j$ and $o_{i j}=0$ if edge $[i, j] \in E$ is 
oriented from $j$ to $i$. The orientation model is as follows:

$$
\begin{array}{rrr}
\operatorname{minimize} & \sum_{[i, j] \in E} c_{i j} \zeta_{i j} & \\
\text { subject to } & f_{j}-f_{i}+\Phi \zeta_{i j} \geq d_{i j} o_{i j}-M\left(1-o_{i j}\right), & \\
& f_{i}-f_{j}+\Phi \zeta_{i j} \geq d_{i j}\left(1-o_{i j}\right)-M o_{i j}, & \forall[i, j] \in E \\
& f_{i} \in \mathcal{F}_{i}, & \forall i \in V \\
& o_{i j}, \zeta_{i j} \in\{0,1\} . & \forall[i, j] \in E
\end{array}
$$

In this formulation, $M$ is an arbitrary, large constant that makes either (2) or (3) active in case $o_{i j}$ is equal to 1 or 0 , respectively. These constraints try to impose the channel separation required for $[i, j] \in E$. In the solution of this orientation model, $o_{i j}=1$ means that the frequency assigned to link $i$ will be smaller than that assigned to link $j$, unless $\zeta_{i j}$ assumes value 1 . Likewise, $o_{i j}=0$ means that the frequency assigned to link $i$ has to be greater than that assigned to link $j$, again unless $\zeta_{i j}$ assumes value 1 . Thus, in this orientation model the variables $o_{i j}$, $[i, j] \in E$, define the set of all orientations of graph $G$.

Notice that we can reformulate the orientation model as follows. For each edge $[i, j] \in E$, let us define a binary variable $o_{j i}$, besides variable $o_{i j}$, such that $o_{i j}=1$ if edge $[i, j]$ is oriented from $i$ to $j$ and $o_{j i}=1$ if edge $[i, j]$ is oriented from $j$ to $i$. According to the new set of orientation variables, we rewrite (2) and (3) as

$$
\begin{array}{ll}
f_{j}-f_{i} \geq d_{i j} o_{i j}-M\left(1-o_{i j}\right), & \forall[i, j] \in E \\
f_{i}-f_{j} \geq d_{i j} o_{j i}-M\left(1-o_{j i}\right) . & \forall[i, j] \in E
\end{array}
$$

Now an orientation of an edge cannot always be defined, but when this happens the channel separation is imposed by one of the new constraints. In order for the objective function to retain its meaning, we must require $\zeta_{i j}=1-o_{i j}-o_{j i}$. It is not difficult to see that a solution to this alternative model is given by an acyclic orientation of a subset of $E$. Additionally, the solution is given by an acyclic orientation of a subset of $E$ with diameter at most $\Phi$ with respect to arc weights $d_{i j}=d_{j i}$.

The orientation model presented in [4] is very similar to the model discussed above, but with another definition for channel separations and, consequently, another definition for the objective function. The approach presented by the authors is based on a two-stage integer programming model in which the outer stage solution is related to an orientation of $G$ with diameter constraints. FAP instances are described with channel separation equal to 1,2 , and 3 , but no computational result is presented. They mention the need for a linear integer formulation for acyclic orientations of diameter at most $\Phi$ with respect to arc weights given by channel separations, and point out some interesting questions to be answered on the structure of the associated polyhedron.

So far we have described how some important, but difficult, combinatorial optimization problems relate to the acyclic orientations of a graph with diameter constraints. The remainder of the paper is devoted to studying a formulation of 
acyclic orientations under binary arc weights. While this can be seen to be immediately useful to the case of vertex coloring and of FAP instances with channel separation equal to 1 , there are no real FAP instances of this type. But channel separations equal to 2 or 3 can also be handled, since we can construct an equivalent, enlarged instance in which all channel separations are 1. Suppose vertices $i$ and $j$ have channel separation $d_{i j}=2$. We define a new vertex $v_{i j}$, new edges $\left[i, v_{i j}\right],\left[j, v_{i j}\right]$ with channel separation equal to 1 , and eliminate the edge $[i, j]$. Additionally to the constraints of the orientation model, it clearly suffices that we introduce the following new constraints: $o_{i v_{i j}}+o_{j v_{i j}} \leq 1$ and $o_{v_{i j}}+o_{v_{i j} j} \leq 1$. An analogous transformation can be done in the cases in which $d_{i j}=3$.

In the next section we enunciate a formulation of acyclic orientations with other path constraints used to describe acyclic orientations with diameter constraints. In the sequel, we refer to the diameter under arc weights that equal 1 simply as diameter.

\section{ACYClic ORIEntations With PATH CONSTRAints}

Let $G=(V, E)$ be an undirected graph with $n=|V|$ vertices and $m=|E|$ edges, and $\kappa$ a positive scalar. We want to describe the acyclic orientations $\omega$ of $G$ that minimize a measure defined over the set of all paths in $G_{\omega}$ with $\kappa$ arcs. Let $D=(V, A)$ be a directed graph with $A=\{(i, j),(j, i) \mid \forall[i, j] \in E\}$. For each arc $(i, j) \in A$, we introduce a binary decision variable $w_{i j}$ such that $w_{i j}=1$ and $w_{j i}=0$ if $\omega([i, j])=(i, j)$, or $w_{i j}=0$ and $w_{j i}=1$ if $\omega([i, j])=(j, i)$. Let $P_{\kappa}(A)$ denote the set of all paths in $D$ with $\kappa$ arcs. Likewise, let $C(A)$ be the set of all cycles in $D$. The formulation follows:

minimize $z$

$$
\begin{array}{lr}
\text { subject to } w_{i j}+w_{j i}=1, & \forall[i, j] \in E \\
\sum_{(i, j) \in C} w_{i j} \leq|C|-1, & \forall C \in C(A) \\
\sum_{(i, j) \in p} w_{i j} \leq z, & \forall p \in P_{\kappa}(A) \\
w_{i j} \in\{0,1\}, & \forall(i, j) \in A \\
0 \leq z \leq \kappa . &
\end{array}
$$

The constraints in (5), (6), and (8) define the acyclic orientations of $G$. Once we have a vector $w \in \mathbb{R}^{2 m}$ satisfying these constraints, let $G_{w}=\left(V, A_{w}\right)$ be the digraph obtained by directing the edges in $E$ according to the variables $w_{i j}$, i.e., a digraph with vertex set $V$ and arc set $A_{w}$ defined as: $(i, j) \in A_{w}$ if and only if $w_{i j}=1$. The variable $z$ is a continuous variable that establishes an upper bound on the overall number of arcs oriented in the same direction in any path of $D$ with at most $\kappa$ arcs. The constraints in (7) and (9) give this meaning to variable $z$. Finally, the objective function in (4) makes variable $z$ assume the minimum possible upper bound. Let us refer to this formulation as $A O(G, \kappa)$. 
The following propositions will guide us in the search for an acyclic orientation with minimum diameter using the above formulation. Recall that $q(G)=\chi(G)-1$.

Proposition 2.1. If $(\bar{w}, \bar{z})$ is a feasible solution to $A O(G, \kappa)$ with $\kappa \leq q(G)$, then $\bar{z}=\kappa$.

Proof. Let $(\bar{w}, \bar{z})$ be a feasible solution of the formulation. Let us assume that $\bar{z}<\kappa=q(G)$. From (7) we conclude that the length of each path in $G_{\bar{w}}$ is less than $q(G)$, i.e., the diameter of $G_{\bar{w}}$ is less than $q(G)$, and we get a contradiction. Thus, $\bar{z} \geq \kappa$. The result follows, since $\bar{z} \leq \kappa$.

Proposition 2.2. If $\left(w^{*}, z^{*}\right)$ is an optimal solution to $A O(G, \kappa)$ with $\kappa \geq q(G)+1$, then $z^{*}<\kappa$.

Proof. Consider an acyclic orientation of $G$ with diameter equal to $q(G)$, and let $(\bar{w}, \bar{z})$ be a solution that induces such an orientation. Let $p$ be a path in $D$ with $\kappa$ arcs. Since $\kappa \geq q(G)+1$ and the diameter of $G_{\bar{w}}$ is $q(G)$, in each succession of $q(G)+1 \operatorname{arcs}$ in $p$ there is at least one arc with $w_{i j}=0$, thus $(\bar{w}, \bar{z})$ with $\bar{z}=\kappa-\lfloor\kappa /(q(G)+1)\rfloor<\kappa$ is a feasible solution of $A O(G, \kappa)$. The result follows, since the objective function of $A O(G, \kappa)$ minimizes the value of $z$.

Now let $U B$ be an upper bound on the minimum diameter $q(G)$ of $G$ and solve $A O(G, \kappa)$ with $\kappa=U B$. If $\left(w^{*}, z^{*}\right)$ is the optimal solution found and $z^{*}=\kappa$, then from Proposition 2.2 it follows that $q(G)=\kappa$. If $z^{*}<\kappa$, then by Proposition 2.1 we can reduce the parameter $\kappa$ by finding the diameter of $G_{w^{*}}$ and assigning its value to $\kappa$. Repeating the entire procedure at most $U B$ times clearly yields an acyclic orientation of $G$ with minimum diameter.

As discussed in Section 1, the solution of some FAP variants can be found by looking for an acyclic orientation with diameter equal to at most a given frequency spectrum $\Phi$. Notice that, if we consider $\kappa=\Phi+1$ and fix $z=\Phi$, then the constraints of $A O(G, \kappa)$ describe the acyclic orientations with diameter at most $\Phi$.

\section{ON THE ACYCLIC SUBGRAPH WITH PATH CONSTRAINTS} POLYTOPE

The acyclic orientation with path constraints polytope, defined as the convex hull of all feasible solutions of the model described in Section 2, is not a fulldimensional one. Let us then consider the following alternative formulation, which models an acyclic subgraph with path constraints:

$$
\begin{aligned}
& \text { minimize } z-(m+1) \sum_{(i, j) \in A} w_{i j} \\
& \text { subject to } w_{i j}+w_{j i} \leq 1, \quad \forall[i, j] \in E \\
& \sum_{(i, j) \in C} w_{i j} \leq|C|-1, \quad \forall C \in C(A) \\
& \sum_{(i, j) \in p} w_{i j} \leq z, \quad \forall p \in P_{\kappa}(A) \\
& w_{i j} \in\{0,1\}, \quad \forall(i, j) \in A \\
& 0 \leq z \leq \kappa \text {. }
\end{aligned}
$$


Notice that (10) allows us to not define an orientation of the edges in $E$. This is where the alternative objective function comes in, since it penalizes any solution that does not orient an edge. It is also clear that all feasible solutions of $A O(G, \kappa)$ are contained in the set of feasible solutions of this new formulation. Moreover, it is not difficult to verify that, if $\left(w^{*}, z^{*}\right)$ is an optimal solution of this formulation, then $w^{*}$ defines an acyclic orientation of $G$ and $z^{*}$ is the smallest value of $z$ satisfying (12). Let us refer to this acyclic subgraph model with path constraints as $A S(G, \kappa)$.

The polytope $P_{G, \kappa}$ associated with $A S(G, \kappa)$ is defined as

$$
P_{G, \kappa}=\operatorname{conv}\left\{(w, z) \in \mathbb{R}^{2 m} \times \mathbb{R} \mid(w, z) \text { satisfies }(10)-(14)\right\} .
$$

Now we turn our attention to the structure of $P_{G, \kappa}$, where we recall that $\kappa>0$. Given an acyclic arc set $B$ of $D$ (i.e., $B \subseteq A$ ), the incidence vector $w^{B} \in \mathbb{R}^{2 m}$ of $B$ is defined as follows: $w_{i j}^{B}=1$, if $(i, j) \in B$, and $w_{i j}^{B}=0$, if $(i, j) \notin B$. Next, the dimension of $P_{G, \kappa}$ is established.

Theorem 3.1. The polytope $P_{G, \kappa}$ is full-dimensional, i.e., $\operatorname{dim}\left(P_{G, \kappa}\right)=2 m+1$.

Proof. Since $P_{G, \kappa}$ contains the null vector, it is sufficient to present other $2 m+1$ linearly independent solutions $(w, z) \in \mathbb{R}^{2 m} \times \mathbb{R}$ in $P_{G, \kappa}$. For each $\operatorname{arc}(i, j) \in A$, let $B_{i j}=\{(i, j)\}$. The $2 m$ solutions $\left(w^{B_{i j}}, 1\right)$, together with the solution $(w, 1)$ with $w$ being the null vector, are clearly linearly independent.

The following theorems establish which constraints of $A S(G, \kappa)$ define facets of $P_{G, \kappa}$.

Theorem 3.2 (Trivial inequalities).

(a) For all $(i, j) \in A, w_{i j} \geq 0$ defines a facet of $P_{G, \kappa}$;

(b) for all $(i, j) \in A, w_{i j} \leq 1$ is not a facet-defining inequality for $P_{G, \kappa}$;

(c) the inequality $z \geq 0$ is not a facet-defining inequality for $P_{G, \kappa}$;

(d) the inequality $z \leq \kappa$ defines a facet of $P_{G, \kappa}$.

Proof. The proof is straightforward.

Notice now that, in $A S(G, \kappa)$, the constraints in (10) can be seen as equivalent to the constraints in (11) with $|C|=2$. We then have the following.

Theorem 3.3 (Cycle inequality). Let $C \in C(A)$. The inequality

$$
\sum_{(i, j) \in C} w_{i j} \leq|C|-1
$$

defines a facet of $P_{G, \kappa}$ if and only if $|C| \leq \kappa$.

Proof. Let $F=\left\{(w, z) \in P_{G, \kappa}\left|\sum_{(i, j) \in C} w_{i j}=\right| C \mid-1\right\}$ be the face of $A S(G, \kappa)$ defined by the cycle inequality written for the cycle $C$. We assume that there is an inequality $a^{T} w+b z \leq c$ valid for $P_{G, \kappa}$ such that $F \subseteq F_{a b}=\left\{(w, z) \in P_{G, \kappa} \mid\right.$ $\left.a^{T} w+b z=c\right\}$ and show that the inequality defining $F_{a b}$ can be written as a 
positive scalar multiple of the cycle inequality defining $F$. Let $\left\{v_{1}, v_{2}, \ldots, v_{|C|}\right\}$ be the vertex set of cycle $C$ and $p_{v_{k} \rightarrow v_{l}}$ the path from vertex $v_{k}$ to vertex $v_{l}$ on $C$. Also, let us assume $v_{|C|+1}=v_{1}$. Consider an $\operatorname{arc}(r, v) \in A \backslash C$. Define an acyclic arc set $B_{1}$ as $B_{1}=p_{v_{k} \rightarrow v_{k-1}}$ if $r=v_{k} \in V(C)$ and $v=v_{l} \in V(C)$, or $B_{1}=p_{v_{1} \rightarrow v_{|C|}}$ in the other cases. Also, define another acyclic arc set $B_{2}=B_{1} \cup\{(r, v)\}$. From the solutions $\left(w^{B_{1}}, \kappa\right)$ and $\left(w^{B_{2}}, \kappa\right)$ in $a^{T} w+b z=c$ we can conclude that $a_{r v}=0$. Now consider the acyclic arc set $B_{3}$ defined as $B_{3}=p_{v_{1} \rightarrow v_{|C|} \mid}$. The solutions $\left(w^{B_{3}}, \kappa\right)$ and $\left(w^{B_{3}}, \kappa-1\right)$ lead to $b=0$. Now we prove the relations among the non-null coefficients in $a^{T} w+b z \leq c$. Suppose $a_{v_{1} v_{2}}=\gamma$. Consider the arc $\left(v_{2}, v_{3}\right)$ and define the arc sets $B_{4}=p_{v_{2} \rightarrow v_{1}}$ and $B_{5}=p_{v_{3} \rightarrow v_{2}}$. The solutions defined by these sets and $z=\kappa-1$ imply $a_{v_{2} v_{3}}=\gamma$. Repeating this argument yields $a_{r v}=\gamma$ for all $(r, v) \in C$. Finally, the cycle inequality defined by a cycle with $|C|>\kappa$ is not a facet-defining one, since in this case constraint $z \leq \kappa$ dominates the cycle inequality.

The methodology to be used in all facet-defining proofs will be the same as in the proof of Theorem 3.3. The following theorem establishes the necessary and sufficient conditions for each inequality in (12) to be facet-defining for $P_{G, \kappa}$.

Theorem 3.4 (Path inequality). Let $p \in P_{\kappa}(A)$ and let $s$ and $t$ be, respectively, the source and sink of $p$. The inequality

$$
\sum_{(i, j) \in p} w_{i j}-z \leq 0
$$

defines a facet of $P_{G, \kappa}$ if and only if $[s, t] \notin E$.

Proof. Let us assume that $\left\{v_{1}, v_{2}, \ldots, v_{\kappa+1}\right\}$ is the set of vertices defining $p$. Consider an $\operatorname{arc}(r, v) \in A \backslash p$ and define an arc set $B_{1}$ as follows: (i) if $r=v_{k}, k \geq 2$, and $v \notin\left\{v_{1}, v_{2}, \ldots, v_{\kappa+1}\right\}: B_{1}=p \backslash\left\{\left(v_{k-1}, v_{k}\right)\right\}$; (ii) if $r \notin\left\{v_{1}, v_{2}, \ldots, v_{\kappa+1}\right\}$ and $v=v_{k}, k \leq \kappa: B_{1}=p \backslash\left\{\left(v_{k}, v_{k+1}\right)\right\}$; (iii) if $r=v_{k}$ and $v=v_{l}, k>l$ : $B_{1}=p \backslash\left\{\left(v_{k-1}, v_{k}\right)\right\}$; (iv) in any other case: $B_{1}=p \backslash\left\{\left(v_{1}, v_{2}\right)\right\}$. Also, define an arc set $B_{2}=B_{1} \cup\{(r, v)\}$. From the solutions $\left(w^{B_{1}}, \kappa-1\right)$ and $\left(w^{B_{2}}, \kappa-1\right)$ we can conclude that $a_{r v}=0$. Now assume $a_{v_{1} v_{2}}=\gamma$ and define the arc sets $B_{3}=p \backslash\left\{\left(v_{1}, v_{2}\right)\right\}$ and $B_{4}=p \backslash\left\{\left(v_{2}, v_{3}\right)\right\}$. The solutions $\left(w^{B_{3}}, \kappa-1\right)$ and $\left(w^{B_{4}}, \kappa-1\right)$ allow us to conclude that $a_{v_{2} v_{3}}=a_{v_{1} v_{2}}=\gamma$. Repeating the same argument we obtain $a_{r v}=\gamma$ for every $\operatorname{arc}(r, v) \in p$. Now consider the arc sets $B_{5}$ and $B_{6}$ defined as $B_{5}=p \backslash\left\{\left(v_{1}, v_{2}\right)\right\}$ and $B_{6}=p$. From the solutions $\left(w^{B_{5}}, \kappa-1\right)$ and $\left(w^{B_{6}}, \kappa\right)$, we conclude that $b=-a_{v_{1} v_{2}}=-\gamma$. Finally, we argue that path constrains cannot be facet-defining when $[s, t] \in E$. Clearly, when $[s, t] \in E$ all solutions satisfying the path inequality with equality also satisfy $w_{t s}=0$.

Next we present new valid inequalities for $P_{G, \kappa}$ which are related to some substructures of $D$. While a cycle inequality is induced by any cycle in $D$, the following theorem introduces another valid inequality induced by cycles with $\kappa+1$ arcs. 
Theorem 3.5 (Cycle- $z$ inequality). Let $C \in C(A)$ be such that $|C|=\kappa+1$. The inequality

$$
\sum_{(i, j) \in C} w_{i j} \leq z
$$

defines a facet of $P_{G, \kappa}$.

Proof. The validity proof is trivial due to (11). The facet-defining proof uses the notation introduced in Theorem 3.3. Also, using arguments analogous to the ones in the proof of Theorem 3.3 we can conclude that $a_{r v}=0$ for every $\operatorname{arc}(r, v) \in A \backslash C$ and that $a_{r v}=\gamma$ for every $\operatorname{arc}(r, v) \in C$. Now consider the $\operatorname{arc} \operatorname{sets} B_{1}$ and $B_{2}$ defined as $B_{1}=p_{v_{1} \rightarrow v_{|C|}}$ and $B_{2}=B_{1} \backslash\left\{\left(v_{1}, v_{2}\right)\right\}$. From the solutions $\left(w^{B_{1}}, \kappa\right)$ and $\left(w^{B_{2}}, \kappa-1\right)$, we conclude that $b=-a_{v_{1} v_{2}}=-\gamma$.

Our next theorem establishes a class of valid inequalities induced by paths with length equal to $\kappa-1$ whose vertices have a common adjacent vertex outside the path.

Theorem 3.6 (Path- $(\kappa-1)$ inequality). Let $p$ be a path in $D$ with $\kappa-1$ arcs and $\left\{v_{1}, v_{2}, \ldots, v_{\kappa}\right\}$ its vertex set. If a vertex $u \in V \backslash\left\{v_{1}, v_{2}, \ldots, v_{\kappa}\right\}$ exists such that $\left[v_{k}, u\right] \in E$ for all $k \in\{1,2, \ldots, \kappa\}$, then the inequality

$$
\sum_{(i, j) \in p} w_{i j}+\sum_{k \in\{1, \ldots, \kappa\}}\left(w_{u v_{k}}+w_{v_{k} u}\right)-\kappa+1 \leq z
$$

is valid for $P_{G, \kappa}$.

Proof. Let $(\bar{w}, \bar{z})$ be a feasible solution in $P_{G, \kappa}$. If $\sum_{k \in\{1, \cdots, \kappa\}}\left(\bar{w}_{u v_{k}}+\bar{w}_{v_{k} u}\right)<\kappa$, then the inequality is trivially satisfied. Assuming this is not the case, the result follows from noticing that, for any feasible orientation of the edges $\left[v_{k}, u\right] \in E$ with $k \in\{1,2, \ldots, \kappa\}$, a suitable path inequality exists involving vertices $u$ and $v_{1}, \ldots, v_{\kappa}$.

Paths in $D$ with $\kappa-2$ arcs also induce valid inequalities for $P_{G, \kappa}$, as the next theorem demonstrates.

Theorem 3.7 (Path- $(\kappa-2)$ inequality). Let $p$ be a path in $D$ with $\kappa-2$ arcs and $\left\{v_{1}, v_{2}, \ldots, v_{\kappa-1}\right\}$ its vertex set. If vertices $u, r \in V \backslash\left\{v_{1}, v_{2}, \ldots, v_{\kappa-1}\right\}$ exist such that $\left[v_{1}, u\right],\left[v_{\kappa-1}, u\right] \in E$ and $[r, u] \in E$, then the inequality

$$
\sum_{(i, j) \in p} w_{i j}+w_{u r}+w_{r u} \leq z
$$

is valid for $P_{G, \kappa}$.

Proof. Let $(\bar{w}, \bar{z})$ be a feasible solution in $P_{G, \kappa}$. If $\bar{w}_{u r}+\bar{w}_{r u}<1$, then the validity of the inequality follows trivially. Assuming this is not the case, the result follows from applying (12) to $p$ extended by $\left\{\left(v_{\kappa-1}, u\right),(u, r)\right\}$ if $\bar{w}_{u r}=1$, and to $p$ preceded by $\left\{(r, u),\left(u, v_{1}\right)\right\}$ if $\bar{w}_{r u}=1$. 
A cycle in $D$ having length $\kappa$ gives rise to yet another class of valid inequalities for $P_{G, \kappa}$.

Theorem 3.8 (Cycle-arcs inequality). Let $C \in C(A)$ be such that $|C|=\kappa$ and $\left\{v_{1}, v_{2}, \ldots, v_{\kappa}\right\}$ be its vertex set. Suppose in addition that $\left\{r_{1}, r_{2}, \ldots, r_{\kappa}\right\} \subset V$ and $A^{\prime}=\left\{e_{1}, e_{2}, \ldots, e_{\kappa}\right\} \subset A$ exist such that:

(i) $\left\{r_{1}, r_{2}, \ldots, r_{\kappa}\right\} \cap\left\{v_{1}, v_{2}, \ldots, v_{\kappa}\right\}=\emptyset$,

(ii) either $e_{k}=\left(r_{k}, v_{k}\right)$ for all $1 \leq k \leq \kappa$ or $e_{k}=\left(v_{k}, r_{k}\right)$ for all $1 \leq k \leq \kappa$.

Then the inequality

$$
\sum_{(i, j) \in C}\left(\lfloor\kappa / 2\rfloor w_{i j}+w_{j i}\right)+\sum_{(i, j) \in A^{\prime}} w_{i j}-\kappa \leq\lfloor\kappa / 2\rfloor z
$$

is valid for $P_{G, \kappa}$.

Proof. Consider the cycle-arcs inequality rewritten as

$$
\lfloor\kappa / 2\rfloor\left(z-\sum_{(i, j) \in C} w_{i j}\right)+\kappa-\sum_{(i, j) \in A^{\prime}} w_{i j} \geq \sum_{(i, j) \in C} w_{j i} .
$$

We first argue that $z-\sum_{(i, j) \in C} w_{i j} \geq 0$; by (11), written for the cycle $C$, an arc $\left(v_{i}, v_{j}\right)$ exists for which $w_{v_{i} v_{j}}=0$, so the latter inequality follows from (12), written for the path $C \backslash\left\{\left(v_{i}, v_{j}\right)\right\} \cup\left\{e_{i}\right\}$. We start with the cases where $z-\sum_{(i, j) \in C} w_{i j}=0$. Since $z=\sum_{(i, j) \in C} w_{i j}$, we can conclude that $\sum_{(i, j) \in A^{\prime}} w_{i j} \leq \sum_{(i, j) \in C} w_{i j}$ (if this were not the case, there would be a path $p$, with $p \cap A^{\prime} \neq \emptyset$, for which (12) would lead to the contradiction of $\left.z>\sum_{(i, j) \in C} w_{i j}\right)$. This observation, together with (10), written for each edge $[i, j]$ such that $(i, j) \in C$, yields

$$
\kappa-\sum_{(i, j) \in A^{\prime}} w_{i j} \geq \kappa-\sum_{(i, j) \in C} w_{i j} \geq \sum_{(i, j) \in C} w_{j i} .
$$

Now we consider the cases where $z-\sum_{(i, j) \in C} w_{i j}=1$. The inequality in (15) is trivially satisfied if $\sum_{(i, j) \in C} w_{j i} \leq\lfloor\kappa / 2\rfloor$. Let us assume that $\sum_{(i, j) \in C} w_{j i} \geq$ $\lfloor\kappa / 2\rfloor+1$. Thus, from (10) and (12) we can conclude that

$$
z \geq\lfloor\kappa / 2\rfloor+1
$$

and

$$
\sum_{(i, j) \in C} w_{i j} \leq \kappa-\sum_{(i, j) \in C} w_{j i} \leq\lfloor\kappa / 2\rfloor .
$$

Notice that the only way to have $z-\sum_{(i, j) \in C} w_{i j}=1$ is to have $\kappa$ odd and also (16) and (17) satisfied with equality. In this case, it follows from satisfying (16) with equality that

$$
\sum_{(i, j) \in C} w_{j i}=z=\lfloor\kappa / 2\rfloor+1 .
$$


From (18) we can conclude that $\sum_{(i, j) \in A^{\prime}} w_{i j} \leq \sum_{(i, j) \in C} w_{j i}$, and from this we arrive at the desired result, since

$$
\kappa-\sum_{(i, j) \in A^{\prime}} w_{i j} \geq \kappa-\lfloor\kappa / 2\rfloor-1=\lfloor\kappa / 2\rfloor .
$$

Finally, in the cases where $z-\sum_{(i, j) \in C} w_{i j} \geq 2$, the inequality in (15) is trivially satisfied, since $\sum_{(i, j) \in C} w_{j i} \leq \kappa-1$.

Notice that the faces defined by the inequalities introduced in Theorems 3.6, 3.7 , and 3.8 are not facets of $P_{G, \kappa}$, since their solutions belong to some face defined by (10). Nevertheless, those inequalities can be computationally useful, since their solutions are feasible solutions to $A O(G, \kappa)$.

Our last theorem establishes that a structure of $G$ constructed from two paths of length $\kappa$ and having some common arcs can induce a valid inequality for $P_{G, \kappa}$.

Theorem 3.9 (Adjacent paths inequality). Let $p^{\mathrm{I}}$ and $p^{\mathrm{II}}$ be paths in $D$ with $\kappa$ arcs. Let $\left\{v_{1}^{\mathrm{I}}, \ldots, v_{\kappa+1}^{\mathrm{I}}\right\}$ and $\left\{v_{1}^{\mathrm{II}}, \ldots, v_{\kappa+1}^{\mathrm{II}}\right\}$ be the respective vertex sets. Suppose $p^{\mathrm{I}}$ and $p^{\mathrm{II}}$ are such that

(i) $v_{k}^{\mathrm{I}}=v_{k}^{\mathrm{II}}$ for $1 \leq k \leq l \leq \kappa, l>1$,

(ii) there exists an edge $\left[v_{r}^{\mathrm{I}}, v_{r}^{\mathrm{II}}\right] \in E$ with $l+1 \leq r \leq \kappa+1$.

Then the inequality

$$
w_{v_{1}^{\mathrm{I}} v_{2}^{\mathrm{I}}}+\sum_{2 \leq k<l} 2 w_{v_{k}^{\mathrm{I}} v_{k+1}^{\mathrm{I}}}+\sum_{l \leq k \leq \kappa}\left(w_{v_{k}^{\mathrm{I}} v_{k+1}^{\mathrm{I}}}+w_{v_{k}^{\mathrm{II}} v_{k+1}^{\mathrm{II}}}\right)+w_{v_{r}^{\mathrm{I}} v_{r}^{\mathrm{II}}}+w_{v_{r}^{\mathrm{II}} v_{r}^{\mathrm{I}}} \leq 2 z
$$

is valid for $P_{G, \kappa}$.

Proof. Consider the path inequalities (12), written for the paths $p^{\mathrm{I}}, p^{\mathrm{II}},\left\{v_{2}^{\mathrm{I}}, \ldots, v_{r}^{\mathrm{I}}\right.$, $\left.v_{r}^{\mathrm{II}}, \ldots, v_{\kappa+1}^{\mathrm{II}}\right\}$, and $\left\{v_{2}^{\mathrm{II}}, \ldots, v_{r}^{\mathrm{II}}, v_{r}^{\mathrm{I}}, \ldots, v_{\kappa+1}^{\mathrm{I}}\right\}$, each with $\kappa$ arcs. Also, consider the inequality $(10)$, written for the edge $\left[v_{r}^{\mathrm{I}}, v_{r}^{\mathrm{II}}\right]$. Adding up these five inequalities and dividing the result by two we obtain

$$
w_{v_{1}^{\mathrm{I}} v_{2}^{\mathrm{I}}}+\sum_{2 \leq k<l} 2 w_{v_{k}^{\mathrm{I}} v_{k+1}^{\mathrm{I}}}+\sum_{l \leq k \leq \kappa}\left(w_{v_{k}^{\mathrm{I}} v_{k+1}^{\mathrm{I}}}+w_{v_{k}^{\mathrm{II}} v_{k+1}^{\mathrm{II}}}\right)+w_{v_{r}^{\mathrm{I}} v_{r}^{\mathrm{II}}}+w_{v_{r}^{\mathrm{II}}} v_{r}^{\mathrm{I}} \leq 2 z+1 / 2 .
$$

The validity of the adjacent paths inequality follows from rounding down the righthand side of the inequality above.

To finalize, we mention that a result similar to Theorem 3.9 also holds if the arcs considered in the statement of the theorem are oriented in the opposite direction.

\section{Concluding Remarks}

We have introduced a formulation of acyclic orientations with path constraints. Our formulation is related to a more general formulation with diameter constraints 
and is, therefore, also related to several combinatorial optimization problems that seek optima on the set of acyclic orientations. We also presented a partial study of the polytope associated with the formulation, introducing further valid inequalities as well.

The results we have presented open up several possibilities for continued research. One of them is to develop separation algorithms that can be used efficiently in a cutting-plane framework. Once results are obtained on this front, a first candidate for a study on applications seems to be the FAP instances with channel separation 1, 2, or 3, as discussed in Section 1. In order to handle the enlarged FAP instances, we first need to develop some preprocessing techniques, such as in [1].

\section{REFERENCES}

[1] K. Aardal, A. Hipolito, C. van Hoesel, B. Jansen, C. Roos, and T. Terlaky. EUCLID CALMA radio link frequency assignment project: A branch-and-cut algorithm for the frequency assignment problem. Technical report, Delft and Eindhoven Universities of Technology, The Netherlands, 1995.

[2] J. Bermond, J. Bond, C. Martin, A. Pekec, and F. Roberts. Optimal orientations of annular networks. Journal of Interconnection Networks, 1:21-46, 2000.

[3] J. Bermond, M. Di Ianni, M. Flammini, and S. Perennes. Acyclic orientations for deadlock prevention in interconnection networks. In Proceedings of the Workshop on Graph-Theoretic Concepts in Computer Science, pages 52-64, 1997.

[4] R. Borndörfer, A. Eisenblätter, M. Grötschel, and A. Martin. The orientation model for frequency assignment problems. Technical Report 98-01, Zuse Institute Berlin, Germany, 1998.

[5] R.W. Deming. Acyclic orientations of a graph and chromatic and independence numbers. Journal of Combinatorial Theory, Series B, 26:101-110, 1979.

[6] T. Gallai. On directed paths and circuits. In P. Erdős and G. Katona, editors, Theory of Graphs, pages 115-118. Academic Press, New York, NY, 1968.

[7] M. Grötschel, M. Jünger, and G. Reinelt. Facets of the linear ordering polytope. Mathematical Programming, 33:43-60, 1985.

[8] M. Grötschel, M. Jünger, and G. Reinelt. On the acyclic subgraph polytope. Mathematical Programming, 33:28-42, 1985.

[9] V. Maniezzo and A. Carbonaro. An ants heuristic for the frequency assignment problem. Future Generation Computer Systems, 16:927-935, 2000.

[10] B. Roy. Nombre chromatique et plus longs chemins d'un graphe. Revue AFIRO, 1:127-132, 1967. 